\title{
Survival after Complete Mesocolic Excision (CME) for Right Sided Colon- cancer Compared to Standard Surgery
}

\author{
Stefan Benz \\ Chefarzt der Klinik für Allgemein-, Viszeral- und Gefäßchirurgie, Kliniken Nagold, Röntgenstraße 20, 72202 Nagold, \\ Germany
}

\begin{abstract}
Purpose: Complete mesocolic excision for right sided colon cancer is a complex operation that might offer a survival benefit. However, studies comparing CME to standard surgery have not been published so far.

Methods: One-hundred-seven patients were operated at the discretion of the surgeon either according to principles of complete mesocolic excision (CME-group, $\mathrm{n}=69$ ) or received a standard right hemicolectomy (non-CME group, $\mathrm{n}=38$ ). The type of surgery was prospectively recorded.

Results: Patients in the non-CME group were significantly older $(67,9$ vs.78,1 $\mathrm{p}<0,001)$ and had fewer adjuvant chemotherapies in stage III $(94,7$ vs. $60,0 \% \mathrm{p}=0,02)$. For the combined analysis of stages I-III actuarial 5 -year overall-, recurrence free- and tumor specific survival was significantly better for the CME group (OS 89,4\% vs. $71,5 \%, \mathrm{p}=0,011$; RFS $83,8 \%$ vs. $70,3 \%$ p $=0,035$; TSS $93,8 \%$ vs. $78,0 \%$ p $=0,049$ ).

Conclusion: The data suggest a survival benefit for complete mesocolic excision in the treatment of right sided colon cancer. However, the data needs to be interpreted with caution because of the uneven distribution of age and number chemotherapies. The results of the large multicentre trial which is currently going on among German certified cancer is expected to further clarify this issue.
\end{abstract}

Keywords: Complete mesocolic excision, CME, colon cancer, right hemicolectomy, D3-lypmhadenectomy.

\section{INTRODUCTION}

In analogy to total mesorectal excision (TME) for rectal cancer complete mesocolic excision was recently introduced by Hohenberger et al. [1] for curative treatment of colon cancer. Like TME CME aims at complete en bloc clearance of the lymphatic drainage of the tumor enveloped in intact fascias of embryologic origin. For left sided Colon cancer this is rather similar to TME, since the same surgical planes are followed. Also, the central vascular ligation (CVL) is equivalent to TME with central transsection of the inferior mesenteric vessels. Therefore, it can be assumed that CME for left sided colon cancer has already been adopted by many surgeons who are familiar with TME. However, for right sided colon cancer CME with CVL is a rather complex operation requiring the dissection of the mesenteric root and the head of pancreas. This has raised some concern about the morbidity risk of this operation. However, CME may have a considerable oncological benefit also in right sided colon cancer. Data concerning morbidity and survival for right sided CME with CVL are scarce, but are urgently required before it can be recommended as routine operation especially considering the frequency of this oncological problem.

Although the concept of CME with CVL was only published in 2009, we have performed right hemicolectomy for colon cancer according to the principles of CME with

*Address correspondence to this author at the Chefarzt der Klinik für Allgemein-, Viszeral- und Gefäßchirurgie, Kliniken Nagold, Röntgenstraße 20, 72202 Nagold, Germany; Tel: 497452 9679301; Fax: 497452 9679301; E-mail: s.benz@klinikverbund-suedwest.de
CVL since 2005 and have prospectively recorded whether the respective surgical radicality was achieved or not. Therefore, we are able to present a series of patients with survival data stratified according to the performance of $\mathrm{CME}$ with CVL.

\section{MATERIALS AND METHODOLOGY}

Since 2005 after informed consent all patients undergoing colorectal resections in our institution were prospectively recorded in a database with 106 items /patient including details of the operation and the postoperative course. Follow-up was updated every 12 months systematically and at any time a patient with recurrent or progressive disease was treated in our hospital. For this paper a retrospective analysis of this database was performed.

For the study presented in this paper, all patients with colon carcinoma in the right hemicolon excluding appendix tumors (ICD 18.0, 18.2, 18.3) were included who were operated between $01 / 01 / 2005$ and 12/31/2010. Surgical radicality was prospectively classified in "not limited" (CME group) and "limited" (non-CME group). The crucial criterion for the classification was the transsection of the ileocolic vein directly at the clearly visualized superior mesenteric vein (VMS). The decision as to which operation was performed was left to the surgeon. Criterions were comorbidity but also training status of the surgeon.

Operation: Other steps of the CME with CVL operation included transsection of the ilecolic artery at the level of the VMS, en-bloc lymphadenectomy of the ventral mesenteric 
a

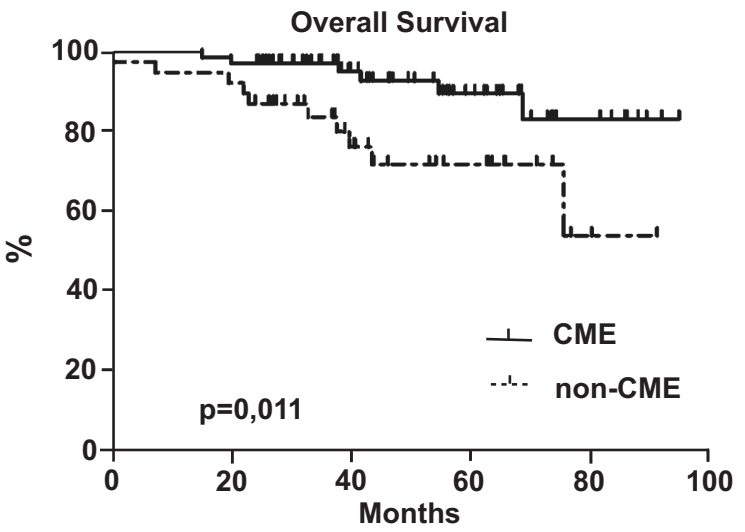

b
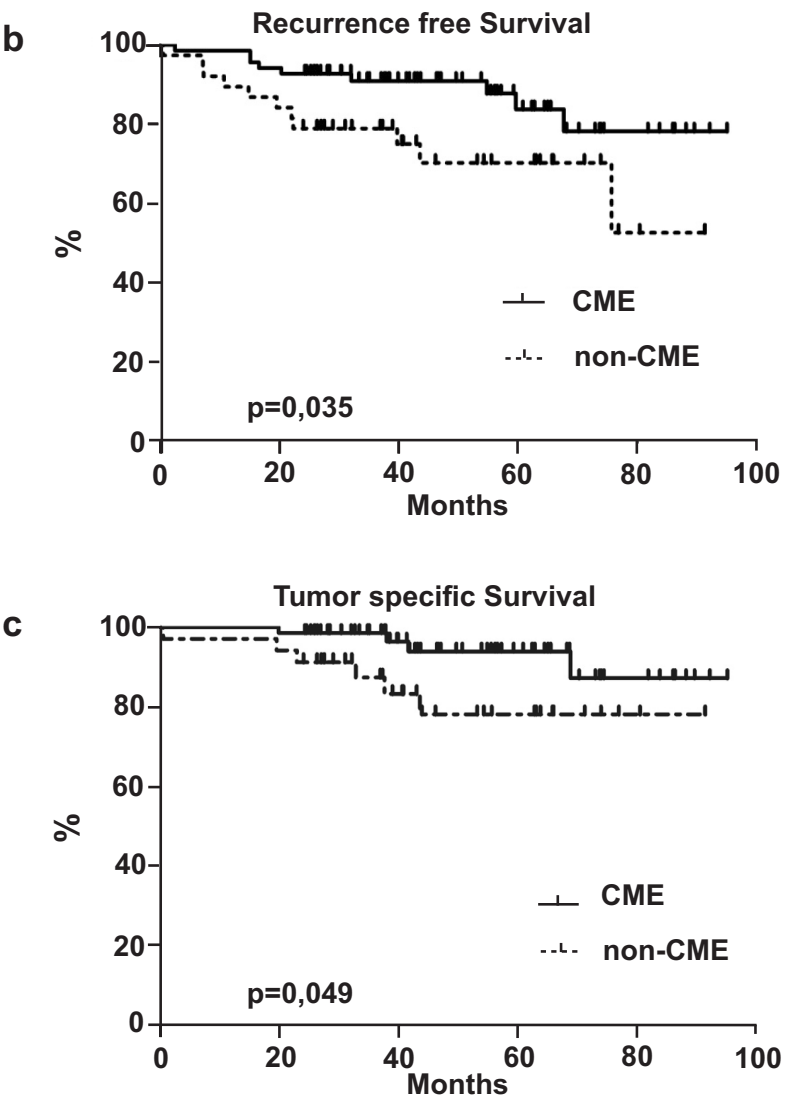

Fig. (1). Survival after CME versus standard surgery.

root anterior to the superior mesenteric artery from approximately $3 \mathrm{~cm}$ distal to the origin of the ilecolic vein to the caudal border of the pancreas. The right branch of the middle colic artery was cut directly at the bifurcation. In carcinomas of the right flexure, the gastroepiploic vessels were resected from the transsection level of the transverse colon to their origin at the pancreatic head and the Henle trunk respectively. In these cases, the middle colic artery was removed completely.

In the non-CME group, the mensenteric root remained untouched and the ileocolic vessels were transsected through the mesocolic "window". Gastroepiploic vessels were left in place.
Statistics: A Survival analysis was performed using the Kaplan-Meier method with log-rank test for comparison as well as calculation of $95 \%$ confidence intervals and hazard ratios (Graphpad prism software). Means were compared using non-parametric U-test, categorical data was compared with Fisher's exact test or chi-square test.

\section{RESULTS}

The present study data from patients with carcinoma in the right hemicolon was used. Fig. (1) shows the selection process for the final analysis.

Patient characteristics are shown in Table 1. As expected from the selection criteria, patients in the non-CME group are significantly older $(67,9$ vs. $78,1 \mathrm{p}<0,001)$. There are also fewer adjuvant chemotherapies in stage III $(94,7$ vs. $60,0 \%$, $\mathrm{p}=0,02$ ) in that group. This might be due to a higher comorbidity in the non-CME group. However, this was not recorded in the database in detail.

Lymph node harvest is markedly higher in the CME group ( 40,1 vs. $29,2 \mathrm{p}=0,001)$. Stage distribution shows no significant overall difference, although there are more stage III patients in the non-CME group ( $27 \%$ vs. $39,5 \%)$. Venous infiltration is more frequently noted in the CME-group (23,2 vs. $5,3 p<0,001)$. All other parameters are evenly distributed. Perioperative morbidity and mortality are equal (Table 2 ). Specific complications related to the dissection of the head of pancreas and the mesenteric vessels did not occur.

Overall survival, recurrence-free survival and tumor specific survival are significantly better in the CME-group compared with the non-CME group for combined analysis of patients in stages I-III (Fig. 2 a-c). When the stages are analyzed separately, it can be noted that the largest contribution for the difference is derived from stage II, and to a lesser extent from stage III. Stage I does not contribute at all (Table 2).

\section{DISCUSSION}

In 1994 and the following years Hermanek et al. [2-4] have characterized for the first time that the huge difference in survival probability in colonic cancer according to the hospital where the operation was performed. He coined the aphorism: The surgeon is the most important risk factor in the treatment of colorectal cancer. However, the factors contributing to this difference were hardly understood. Surgical radicality seemed not to make the crucial difference since Rouffet [5] could not show any difference between segmental resections and hemicolectomies. However, in this only randomized trail dealing with surgical radicality in colon cancer the quality of surgery in respect to lymph node counts, the amount of mesentery removed and the intactness of the mesocolic fascias was not reported.

In contrast to this finding, the number of evaluated lymph nodes are reported to strongly determine the risk of recurrence in UICC II patients in numerous studies [6, 7], implying an advantage for more radical surgery. In addition, mobilization of the colon respecting embryological planes was reported to confer a survival benefit [8]. Furthermore, the development of TME surgery in the last 20 years pointed to an increase in surgical radicality in the treatment also of 
Table 1. Patient Characteristics and Tumor Stage

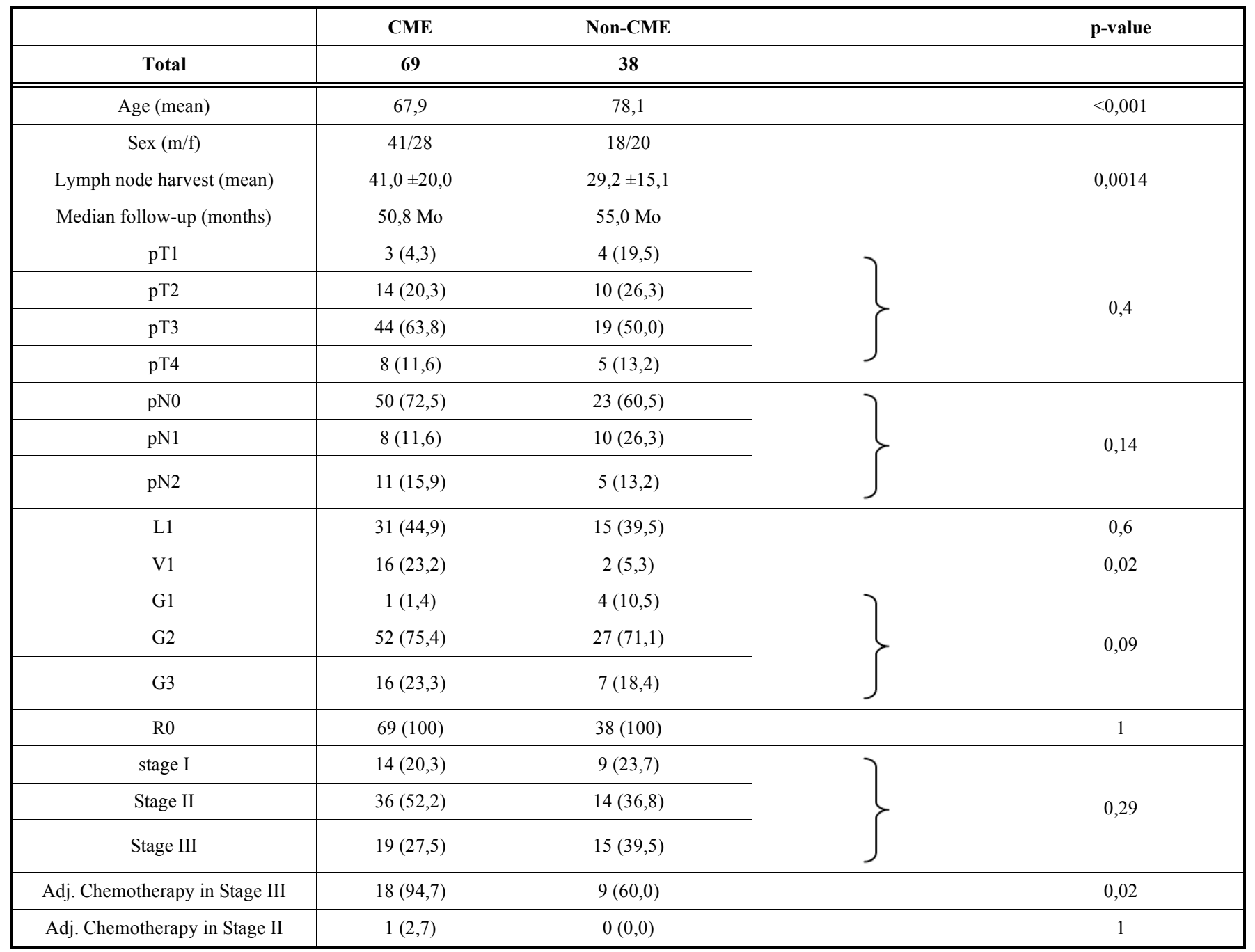

Numbers in brackets represent percentages

Table 2. Survival Data by Stage and Perioperative Morbidity

\begin{tabular}{|c|c|c|c|c|}
\hline CME & \multicolumn{2}{|c|}{ Non-CME } & p-Value & HR (95\%KI) \\
\hline Stage I & 100 & 100 & 1 & \\
\hline Stage II & 88,5 & 62,5 & 0,015 & $0,14(0,02-0,69)$ \\
\hline Stage III & 83,7 & 62,2 & 0,14 & $0,34(0,081-1,45)$ \\
\hline Stage I & 100 & 100 & 1 & \\
\hline Stage II & 85,9 & 62,5 & 0,04 & $0,20(0,046-0,93)$ \\
\hline Stage III & 72,2 & 60 & 0,23 & $0,47(0,13-1,6)$ \\
\hline Stage I-III & 83,8 & 70,3 & 0,035 & $0,36(0,14-0,93)$ \\
\hline Stage III & 83,7 & 66,5 & 0,26 & $0,41(0,08-1,94)$ \\
\hline
\end{tabular}


Table 2. contd....

\begin{tabular}{|c|c|c|c|c|}
\hline CME & \multicolumn{2}{|c|}{ Non-CME } & p-Value & HR (95\%KI) \\
\hline \hline Stage I-III & 93,8 & 78 & 0,049 & $0,26(0,06-0,99)$ \\
\hline Perioperative Mortality & $0 / 69$ & $1 / 38$ & 1 & 1 \\
\hline Relaparotomy & $5 / 69$ & $2 / 38$ & 1 & \\
\hline
\end{tabular}

\section{Situs after CME surgery}

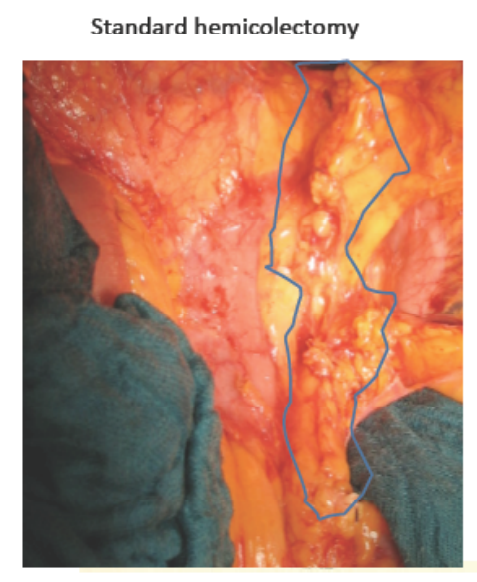

(a)

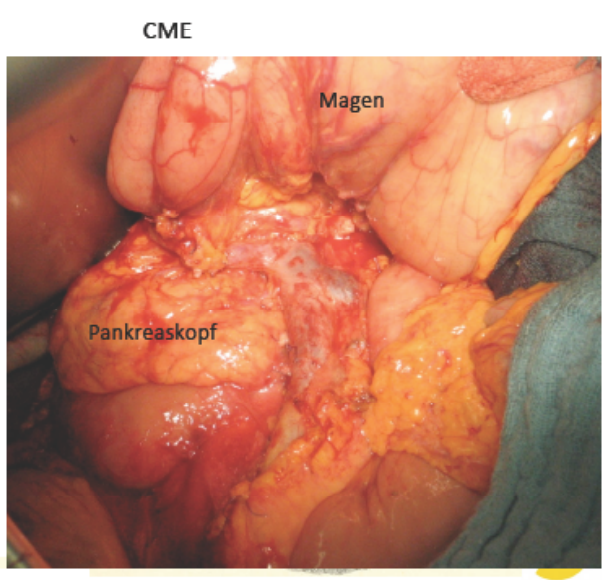

(b)

\section{Specimen after CME surgery}

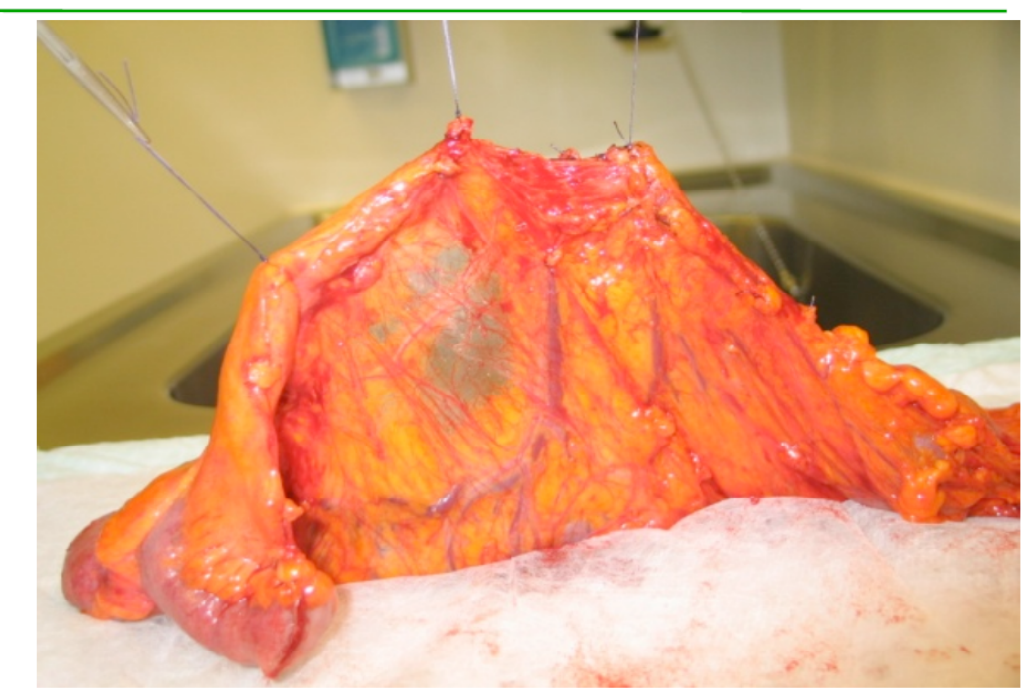

(c)

Fig. (2). After standard surgery the mesenteric vessels and the pancreatic head remain covered by fatty and lymphatic tissue respectively (a). In contrast, after CME surgery the ventral aspect of the mesenteric root and the pancreatic head is cleared from all lymphatic tissue (b). The specimen after CME contains the lymphatic tissue of the mesenteric root. The dorsal aspect of the specimen has a shiny smooth surface made up by the intact mesocolic fascia (c).

colon cancer [9]. Thus, an analogous operation to TME in which extensive surgical radicality is combined with subtle treatment of the mesocolon in order to produce a specimen with intact mesocolic layers was developed for the colon. The European variant of the operation is the complete mesocolic excision with central vascular ligation first described by Hohenberger, the Japanese variant is the D3 Resection [10]. Using this operation, very good results have been reported in retrospective series depending on the amount of mesentery removed and the surgical plane 
followed [11-13]. However, a prospective comparison between CME and standard surgery for right sided colon cancer has not been done so far.

The 5 year overall survival of $89.4 \%$ reported for the $\mathrm{CME}$ group in this paper is very high and is comparable with the data published by Hohenberger [1] for CME surgery. In addition, 5-year survival of $71.5 \%$ in the non- CME group is within a close range of the data published by Kube for a German quality assurance program including 31055 patients. The SEER database reports even lower survival rates [14]. Therefore, the effect for CME surgery demonstrated here compares well to the data published so far. Also most importantly, perioperative morbidity and mortality did not differ between the groups, although the lack of statistical power for this statement has to be considered.

What are the limitations of the study? First, the decision whether to perform CME with CVL or not was not randomized. It was dependent on the surgeon`s training (in 2005 one of three surgeon's performed CME in 2010 two of three) and the comorbidity of the patient. The difference in age of the groups influences the results of overall-survival and recurrence-free survival. However, tumor specific survival clearly shows that most deaths are attributable due to malignant disease. A further important shortcoming is the imbalance of chemotherapies in stage III. But if survival in the separate stages is looked at, it becomes obvious that the major contribution to the effect is derived from stage II. Another limitation is that the stratification in CME and nonCME was only based on one criterion, (transection of the ileocolic vein flush with and under clear visualization of the VMS). However, it is noteworthy that this classification resulted in specimen with significantly different lymph node yields in clearly distinctive surgical radicality. A further limitation is that only surgical radicality was recorded prospectively. The other main principle of CME -the intactness of the resected mesentery-, was only classified and recorded since 2009 and could therefore not be included in this analysis. But it is clear, that if a surgeon has adopted the principles of CME surgery respecting the planes, he will use this technique irrespective of the intended radicality of the operation. This behavior might have diminished the effect in survival difference. Also, the number of patients included in the study is fairly low and there is a considerable number of "lost of follow-ups". On the other hand, only tumors of the right hemicolon are included, which ameliorates comparability especially of technical aspects.

\section{CONCLUSION}

This study confirms the excellent survival for patients with right sided colon cancer treated by CME with CVL, and suggests a benefit compared to standard surgery without increasing morbidity and mortality. However, confirmation of these data by a large multicentre trial is necessary and is currently underway (Resektatstudie) in German certified colorectal cancer centers.

\section{CONFLICT OF INTEREST}

The authors confirm that this article content has no conflicts of interest.

\section{ACKNOWLEDGEMENTS}

The work of Mrs. Alexandra Gayer for managing the database and performing the follow-up is gratefully acknowledged.

\section{REFERENCES}

[1] Hohenberger W, Weber K, Matzel K, et al. Standardized surgery for colonic cancer: complete mesocolic excision and central ligation--technical notes and outcome. Colorectal Dis 2009; 11(4): 354-64

[2] Hermanek $\mathrm{P}$, Wiebelt $\mathrm{H}$, Riedl $\mathrm{S}$, et al. Long-term results of surgical therapy of colon cancer. Results of the Colorectal Cancer Study Group. Chirurg 1994; 65(4): 287-97.

[3] Hermanek P, Mansmann U, Altendorf-Hofmann A, et al. Comparative study of oncological outcome quality in colorectal carcinoma--ranking by surrogate endpoint? Chirurg 1999; 70(4): 407-14.

[4] Hermanek P Impact of surgeon's technique on outcome after treatment of rectal carcinoma. Dis Colon Rectum 1999; 42(5):55962.

[5] Rouffet F, Hay JM, Vacher B, et al. Curative resection for left colonic carcinoma: hemicolectomy vs. segmental colectomy. A prospective, controlled, multicenter trial. French Association for Surgical Research. Dis Colon Rectum 1994; 37(7): 651-9.

[6] Le Voyer TE, Sigurdson ER, Hanlon AL, et al. Colon cancer survival is associated with increasing number of lymph nodes analyzed: a secondary survey of intergroup trial INT-0089. J Clin Oncol 2003; 21(15): 2912-9.

[7] Chang GJ, Rodriguez-Bigas MA, Skibber JM, et al. Lymph node evaluation and survival after curative resection of colon cancer: systematic review. J Natl Cancer Inst 2007; 99(6): 433-41.

[8] Bokey EL, Chapuis PH, Dent OF, et al. Surgical technique and survival in patients having a curative resection for colon cancer. Dis Colon Rectum 2003; 46(7):860-6.

[9] Martling A, Holm T, Rutqvist LE, et al. Impact of a surgical training programme on rectal cancer outcomes in Stockholm. Br J Surg 2005; 92(2): 225-9.

[10] West NP, Kobayashi H, Takahashi K, et al. Understanding optimal colonic cancer surgery: comparison of Japanese D3 resection and European complete mesocolic excision with central vascular ligation. J Clin Oncol 2012; 30(15): 1763-9.

[11] West NP, Hohenberger W, Finan PJ, et al. Mesocolic plane surgery: an old but forgotten technique? Colorectal Dis 2009; 11(9): 988-9.

[12] West NP, Hohenberger W, Weber K, et al. Complete mesocolic excision with central vascular ligation produces an oncologically superior specimen compared with standard surgery for carcinoma of the colon. J Clin Oncol 2010; 28(2): 272-8.

[13] West NP, Morris EJ, Rotimi, O et al. Pathology grading of colon cancer surgical resection and its association with survival: a retrospective observational study. Lancet Oncol 2008; 9(9):857-65.

[14] Paulson EC, Mitra N, Sonnad S et al. National Cancer Institute designation predicts improved outcomes in colorectal cancer surgery. Ann Surg 2008; 248(4): 675-86. 